\title{
Developing a timeline for evaluating public health nutrition policy interventions. What are the outcomes and when should we expect to see them?
}

\author{
Jennie I Macdiarmid ${ }^{1, *}$, Jennifer Loe ${ }^{1}$, Flora Douglas ${ }^{2}$, Anne Ludbrook ${ }^{3}$, Caroline \\ Comerford ${ }^{4}$ and Geraldine McNeill ${ }^{1,2}$ \\ 'Public Health Nutrition Research Group, Rowett Institute of Nutrition and Health, University of Aberdeen, \\ Greenburn Road, Bucksburn, Aberdeen AB2 1 9SB, UK: ${ }^{2}$ Population Health, University of Aberdeen, Aberdeen, \\ UK: ${ }^{3}$ Health Economics Research Unit, University of Aberdeen, Aberdeen, UK: ${ }^{4}$ NHS Grampian, Aberdeen, UK
}

Submitted 13 January 2010: Accepted 21 June 2010: First published online 16 August 2010

\begin{abstract}
Objective: To develop a timeline for evaluating public health nutrition policy interventions.

Design: Concept mapping, a stakeholder-driven approach for developing an evaluation framework to estimate the 'time to impact' for policy interventions. The Schools (Health Promotion and Nutrition) (Scotland) Act 2007 was used as the model to develop the evaluation timeline as it had typical characteristics of government policy. Concept mapping requires stakeholders to generate a list of the potential outcomes, sort and rate the outcomes. Multidimensional scaling and hierarchical cluster data analysis were used to develop an anticipated timeline to impact for the policy.

Setting: United Kingdom.

Subjects: One hundred and eleven stakeholders representing nutrition, public health, medicine, education and catering in a range of sectors: research, policy, local government, National Health Service and schools.

Results: Eighty-five possible outcomes were identified and grouped into thirteen clusters describing higher-level themes (e.g. long-term health, food literacy, economics, behaviour, diet, education). Negative and unintended consequences were anticipated relatively soon after implementation of the policy, whereas positive outcomes (e.g. dietary changes, health benefits) were thought likely to take longer to emerge. Stakeholders responsible for implementing the legislation anticipated that it would take longer to observe changes than those from policy or research.

Conclusions: Developing an anticipated timeline provides a realistic framework upon which to base an outcome evaluation for policy interventions and identifies positive and negative outcomes as well as considering possible unintended consequences. It offers benefit to both policy makers and researchers in mapping the progress expected towards long-term health goals and outcomes.
\end{abstract}

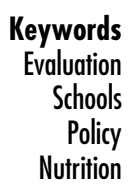

There is increasing demand for evaluation of public health policy to demonstrate effectiveness ${ }^{(1-3)}$. Such policies, however, tend to be complicated with multiple interacting components, complex partnerships and external factors which have the potential to reduce the impact of the intervention, and this provides a challenge for evaluation $^{(4-6)}$. Unlike most clinical studies, the theory underpinning a policy and the specific aims and objectives are not always clear. Issues associated with evaluating policy interventions are still debated ${ }^{(5)}$, but it is increasingly recognised that a different approach from that for clinical studies is needed ${ }^{(7-10)}$. Often a wide range of strategically chosen outcomes need to be identified which are consistent with and sensitive to the purpose of the intervention ${ }^{(6,11,12)}$. A single outcome may not give adequate assessment of the policy intervention, or may ask too much of the intervention ${ }^{(13,14)}$. Realistic expectations are also needed for what policy interventions can deliver within a specified time frame, particularly as they often involve a process of social change ${ }^{(15,16)}$.

Several different methods have been used to try to conceptualise this complexity (e.g. logic models ${ }^{(17,18)}$, concept mapping $\left.{ }^{(19)}\right)$, with a common aim to identify short-, medium- and long-term outcomes, showing a 
Table 1 Summary of the main principles of the Schools (Health Promotion and Nutrition) Act (Scotland) 2007

Places health promotion at the heart of a school's activities

Ensures that food and drink served in schools (i.e. school lunches, breakfast and snacks at any other time of the day) meet nutritional requirements specified by the Scottish Ministers by regulations

Ensures local authorities promote the uptake and benefits of school meals and, in particular, free school meals

Reduces the stigma associated with free school meals by requiring local authorities to protect the identity of those eligible for free school meals

Gives local authorities the power to provide pupils with healthy snacks and drinks, either at a cost or free of charge

Requires local authorities to consider sustainable development guidance when they provide food or drink in schools

More detailed information about the Act can be found on the Scottish government website (www.scotland.gov.uk/Topics/Education/Schools/HLivi/foodnutrition).

logical progression of change. Concept mapping, which brings together knowledge and experiences of stakeholders to develop a common framework for evaluation $^{(19)}$, is particularly useful for long-term programmes, such as public health policies, where there is limited theoretical and empirical knowledge of the time to impact. The estimated time for a policy intervention to have a measureable impact is often not specified, which can lead to unrealistic expectations of the outcome and the issue of whether it has failed or if the outcomes have simply not occurred yet ${ }^{(13,20,21)}$. Concept mapping has been used in the evaluation and planning of a number of public health policies (e.g. evaluation of the Smoking, Health and Social Care (Scotland) Act 2005, planning health improvement initiatives ${ }^{(22)}$ and determining intervention strategies to increase physical activity ${ }^{(23)}$ ).

Despite raised awareness of diet and health and the number of community-based interventions aimed at improving dietary intakes, there is a lack of well-planned evaluations $^{(2,24)}$. The UK House of Commons Health Committee $^{(3)}$ recently highlighted this, stating that 'even where evaluation is carried out, it is usually "soft", amounting to little more than examining processes and asking those involved what they thought about them'.

The aim of the project described here was to develop an evaluation framework with a timeline for assessing the impact of nutrition-related policy interventions; the development used the implementation of the Schools (Health Promotion and Nutrition) (Scotland) Act $2007^{(25)}$ in primary schools since it is an example of typical government policy, but the intention was that the framework would have wider relevance. The Act applies to both primary and secondary schools but the focus for the project was primary schools (pupils aged 5-11 years). The legislation covers a range of health promotion issues as well as setting regulations for all food and drink provided in schools to meet nutritional standards. The main principles of the Act are shown in Table 1; more detailed information (e.g. the nutrient regulations, restriction of specific food and drinks, and the health promotion within schools) can be found on the Scottish government website (www.scotland.gov.uk/Topics/Education/Schools/ HLivi/foodnutrition). This policy is similar to others being implemented in other countries and is typical of many public health policies which have a number of objectives and actions within a single policy ${ }^{(26,27)}$. There was also sufficient detail to allow stakeholders with a range of expertise and prior knowledge of the Act to participate.

\section{Methods}

\section{Participants}

Concept mapping is a stakeholder-driven method. Stakeholders from scientific research, national and local government, the National Health Service (NHS), schools, community organisations and parents with children in primary school participated in the study. This wide range of stakeholders collectively had expert knowledge of the dayto-day schools context, research knowledge associated with schools-based health promotion, behaviour change and nutrition issues, as well as knowledge on policy processes and their intended aims. Stakeholder participation varied at different stages of the project, as described in subsequent sections. Key stakeholders were sent a written invitation to participate in the sorting and rating stage of the project ( $n$ 291), with additional invitations sent out via national and local nutrition organisations in the UK (Food and Health Alliance, The Nutrition Society, British Dietetic Association, Scottish Colloquium on Food and Feeding).

\section{Concept mapping process}

Concept mapping is a mixed-methods approach, combining qualitative research methods with multivariate statistical analyses, identifying and grouping key outcomes of an intervention. The process has six stages: (i) defining the evaluation question; (ii) brainstorming the possible outcomes; (iii) sorting and rating the outcomes; (iv) data analysis; (v) interpretation of the results; and (vi) development of a timeline.

\section{Evaluation question}

The aim of the project was to identify the likely outcomes resulting from the implementation of the Schools Act in primary schools. The following statement was developed for stakeholders to complete in the brainstorming session: 'I think the introduction of the Schools (Health Promotion and Nutrition) (Scotland) Act 2007 in primary schools will ...'

\section{Brainstorming: generate outcome statements}

Three methods were used to generate outcome statements: a workshop, semi-structured interviews and focus 
groups. The workshop was run with nineteen stakeholders from research and policy sectors across Scotland. Following a presentation describing in detail the Schools Act and purpose of the workshop, participants were asked to individually generate between six and eight outcomes that completed the statement 'I think the introduction of the Schools (Health Promotion and Nutrition) (Scotland) Act 2007 in primary schools will ...', then to work in groups to agree and refine the list of statements. The groups included a mix of stakeholders from different sectors, with varying prior knowledge of the Act. Statements from all the groups were collated. Semi-structured interviews were carried out with stakeholders from local government departments involved with the implementation of the Schools Act (education ( $n$ 2), catering $(n 3)$ and health $(n 4))$ in Scotland. Interviewees described their role and previous experience of nutrition and health promotion policies in schools and were asked to describe what they thought would be the likely outcomes from the implementation of the Act. Interviews were transcribed and statements describing the outcomes extracted independently by two researchers (J.I.M., J.L.) and then combined. The opinions of parents with children in primary school were explored through two focus groups (six parents per group). The discussion focused on their views of school lunches and health promotion in schools, their knowledge of the Schools Act and the changes that they anticipated with the implementation of the Act.

\section{Structuring: sorting and rating the statements}

All the statements from the workshop, interviews and focus groups were combined (removing duplications) and reviewed both internally and externally, reducing the final list to eighty-five possible outcomes. A larger group of stakeholders was then sent information about the project (including the concept mapping methodology), a detailed description of the Schools Act and instructions for completing the sorting and rating. They were asked to first sort the statements into groups based on the similarity between statements in a way that made sense to them. Each statement could be placed in only one group and the statements could not all be put in a single group or grouped simply as positive or negative, but had to be grouped into themes. Next they rated each of the statements in terms of the relative likelihood of the outcome occurring, the importance of the outcome and the anticipated earliest time to measureable impact. Both the likelihood and importance were rated on a 5-point Likert scale from 1 (not at all) to 5 (extremely). Time to impact was rated on a 7-point scale with yearly intervals from 0 (immediate) to 6 (6 years or more). The sorting and rating exercise was completed online using the Concept System Global software (Concept Systems Inc., Ithaca, NY, USA), enabling a larger, more geographically dispersed group to participate. Stakeholders provided background information on age, sex, the type of sector they worked in, their area of expertise, their knowledge of and role in the Schools Act and whether they had children in primary school.

\section{Representation: data analysis}

Individual matrices were generated from the sorted data showing groupings by individuals, which were then aggregated into a similarity matrix to show the number of participants who sorted each pair of statements together. Using multidimensional scaling (MDS), two-dimensional coordinates were created for each statement on the concept map to give a visual representation of the data. The distance between the points (i.e. statements) on the map represents the similarity between the statements, with those sorted together by more of the participants being closer together. The stress value was calculated for the MDS as a measure of the goodness-of-fit of the map (lower values indicate a better fit). Hierarchical cluster analysis was performed using the coordinates produced by the MDS to group the statements. An iterative process reducing the number of clusters from an initial twenty clusters down to eight was examined for interpretability. Data from fifteen stakeholders were excluded because they had not grouped the statements according to the instructions described above. Data were analysed using the Concept System software.

The average likelihood, importance and time to impact ratings were calculated for each statement and each cluster. The average rating for each cluster was compared between stakeholder groups using general linear models in the SPSS statistical software package version $17 \cdot 0$ (SPSS Inc., Chicago, IL, USA). The groups were based on their type of work: research (academic researchers with knowledge of scientific studies, $35 \%$ ); policy (national government or government organisations involved in policy making, 19\%); and implementation (local government, NHS, schools and community groups responsible for implementing legislation, $44 \%$ ).

\section{Interpretation: development of the timeline}

The average rating of the anticipated time to impact for each cluster was used to develop the timeline. Time to impact ratings for individual statements were excluded if rated as 'not at all' likely to occur, as it was considered to be meaningless if the participant thought it would not occur. The number excluded per statement ranged from 0 to 35 ratings (median per statement, 3 ).

\section{Results}

\section{Participant characteristics}

Table 2 shows the characteristics of the stakeholders who completed the online rating exercise ( $n$ 102). It was not possible to estimate the exact response rate since the 
Table 2 Characteristics of the stakeholders who completed the online sorting and rating exercise

\begin{tabular}{|c|c|c|c|c|}
\hline & \multicolumn{4}{|c|}{ Sector } \\
\hline & All $(n$ 102) & Research ( $n$ 36) & Policy (n 19) & Implementation ( $n$ 45) \\
\hline Female (\%) & 85 & 86 & 84 & 84 \\
\hline \multicolumn{5}{|l|}{ Sector $(\%)$} \\
\hline University/research institutes & 35 & 100 & - & - \\
\hline National government & 19 & - & 100 & - \\
\hline Local government & 17 & _ & - & 40 \\
\hline National health service & 18 & - & - & 40 \\
\hline Schools & 8 & - & - & 18 \\
\hline Community organisations & 1 & - & - & 2 \\
\hline Other & 2 & - & - & - \\
\hline \multicolumn{5}{|l|}{ Background (\%) } \\
\hline Nutrition & 37 & 47 & 47 & 27 \\
\hline Health promotion & 13 & 3 & 11 & 22 \\
\hline Policy & 3 & 3 & 11 & 0 \\
\hline Catering & 12 & 0 & 0 & 27 \\
\hline Education & 10 & 0 & 5 & 20 \\
\hline Social sciences & 11 & 30 & 5 & 0 \\
\hline Economics & 1 & 0 & 5 & 0 \\
\hline Evaluation & 4 & 3 & 16 & 0 \\
\hline Medicine/physiology & 4 & 11 & 0 & 0 \\
\hline Other & 2 & 3 & 5 & 4 \\
\hline \multicolumn{5}{|l|}{ Knowledge of the Schools Act (\%) } \\
\hline None & 10 & 25 & 0 & 0 \\
\hline Small amount of detail & 12 & 25 & 5 & 5 \\
\hline Some detail & 35 & 39 & 37 & 31 \\
\hline Lots of detail & 43 & 11 & 58 & 64 \\
\hline \multicolumn{5}{|l|}{ Role played in the Schools Act (\%) } \\
\hline None & 38 & 67 & 26 & 18 \\
\hline Development & 9 & 3 & 42 & 0 \\
\hline Implementation & 29 & 0 & 0 & 67 \\
\hline Monitoring & 3 & 3 & 5 & 2 \\
\hline Research relating & 9 & 17 & 16 & 0 \\
\hline Other & 12 & 10 & 11 & 13 \\
\hline
\end{tabular}

authors did not have access to the number of additional invitations emailed via professional societies. One hundred and fifty-four stakeholders signed up to the website and 111 completed the sorting (72\%); 102 completed all three sets of ratings $(66 \%)$.

\section{Concept maps}

Over 300 statements were generated, many of these were duplications and the list was reduced to eighty-five unique statements. From the cluster analysis a consensus was reached that the thirteen cluster option provided logical groupings. Positioning of the statements and clusters is shown on the concept map (Fig. 1), with clusters comprising between three and thirteen statements. The stress value for the MDS was $0 \cdot 251$, which was comparable with previous studies $\left(0 \cdot 205\right.$ to $\left.0 \cdot 365^{(28)}\right)$.

The clusters included positive and negative aspects of health, diet, knowledge, social norms and economics (Table 3). Nine clusters were positive outcomes, i.e. improvements in the uptake of school meals, diet and food intake, healthy dietary choices, food literacy, food culture, local economy, educational benefits, long-term health and well-being. Four clusters were negative or unintended consequences, i.e. undesirable eating behaviours, adverse economic consequences, negative attitudes and behaviours and a range of unintended consequences (e.g bullying, food waste, reduction in family meals together).

\section{Ratings by cluster}

'Increase food literacy' (cluster 4) was rated as the most likely outcome, followed by 'improve school meals and uptake' (cluster 7) and 'improve food culture' (cluster 5). Negative and unintended consequences (clusters 11, 12 and 13) were rated as less likely to occur. Clusters tended to have higher ratings for importance than likelihood, with the exception of 'adverse economic consequences' (cluster 10). Figure 2 shows the relationship between importance and likelihood ratings for individual statements.

\section{Timeline}

Using the average earliest anticipated time for impact for each cluster, it was possible to identify short-, mediumand long-term outcomes (Fig. 3). The arrows intercepting the timeline ( $x$-axis) represent the average rating for each cluster. The width of each box is set by the minimum and maximum rating for individual statements within the cluster. For example, in cluster 1 the average time to impact was $5 \cdot 02$, ranging from $4 \cdot 22$ (dental health) to $5 \cdot 71$ (health of the general population and reduce costs to 


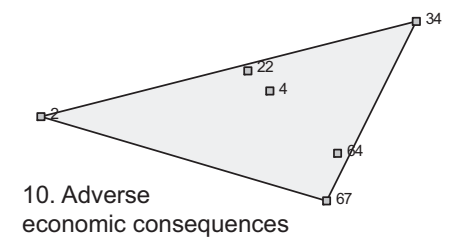

economic consequences

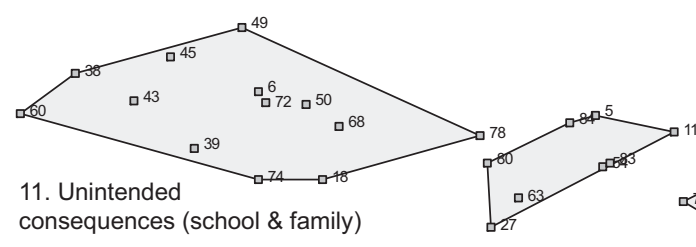

consequences (school \& family)

12. Negative effect on attitudes \& behaviour

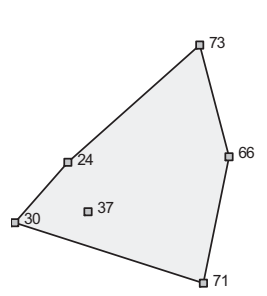

9. Improve local food economy
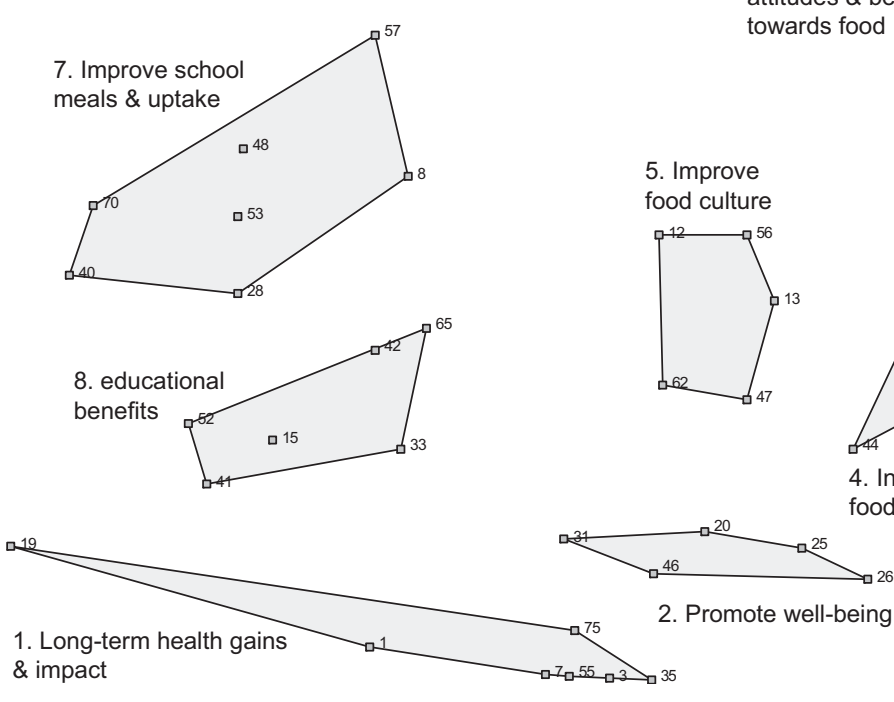

Fig. 1 Concept map (the numbers in the clusters relate to the statements in Table 3)

the NHS). The timeline serves to illustrate realistic expectations for change. Although there is a clear order for the clusters based on the average time to impact there is considerable overlap between them. The timeline does not serve as a precise single time point to assess change but a realistic guide for approximate estimates when change would be anticipated. Different levels of shading of the clusters in Fig. 3 illustrate the likelihood of the outcome, with darker shading illustrating a higher likelihood.

\section{Time to impact by stakeholder group}

The anticipated time to impact for each of the clusters was compared between stakeholder groups. A significant difference in the anticipated time to impact was found between groups for all of the clusters except clusters 1 ('long-term health gains and impact'), 11 ('unintended consequences in school and on the family') and 13 ('undesirable eating behaviours'; Table 4). Post hoc tests showed significant differences between the implementation and research groups for clusters 2, 3, 4, 5, 6, 7, 8 and 12 and between the implementation and policy groups for clusters $4,5,7,8,9$ and 10. In all cases the implementation group anticipated that the time to impact would be longer. The research and policy groups did not differ significantly on average time to impact for any of the clusters. Detailed prior knowledge about the Schools Act varied between groups, $11 \%$ for the research group, $58 \%$ for the policy group and $65 \%$ for the implementation group, but adjusting for this did not alter the differences found between the groups.

\section{Discussion}

A systematic process was used to conceptualise and develop a timeline for evaluating nutrition policy in schools. This analysis grouped a complex range of behavioural, socio-cultural, health and economic outcomes into clusters and estimated realistic expectations of the time lag between implementation and impact on the range of outcomes. A common problem in evaluating public health policy is that programme theories (if stated) seldom specify the length of time for observed behavioural changes and long-term health gains to emerge, which can lead to unrealistic expectations $^{(13,21)}$. In the present study, stakeholders anticipated that on average it would take over 5 years to detect any measureable change in long-term health outcomes (e.g. obesity, dental health and diabetes), which was 2 years longer than when they anticipated dietary changes. This presents a huge challenge for both policy makers and researchers trying to determine the impact of a long-term policy within a relatively short policy cycle. This highlights the importance of short- and intermediate-term outcomes as proximal indicators of whether the policy intervention is likely to produce the desired long-term outcome. Furthermore, initial success can often create the conditions for further success; for example, children need to try a new food, made available to them through school meals, before they decide if they like it and subsequently include it in their diet or not, and it is important to monitor these small changes. 
Table 3 Statements included in each of the thirteen clusters and relative ratings of likelihood and importance

\begin{tabular}{|c|c|c|}
\hline & Likelihood & Importance \\
\hline Cluster 1: LONG-TERM HEALTH GAINS \& IMPACT & $3 \cdot 03$ & $4 \cdot 16$ \\
\hline
\end{tabular}

1. Improve the health of the general population

3. Increase the number of children with a healthy weight

7. Reduce the prevalence of overweight and obesity

35. Lower blood pressure and cholesterol levels among children

55. Reduce the prevalence of diabetes in children (type 2)

75. Improve dental health

19. Reduce costs to the National Health Service

Cluster 2: PROMOTE WELL-BEING

20. Encourage other healthy lifestyle choices

25. Help children achieve the Scottish dietary targets

26. Improve the micronutrient status of children

31. Improve self-esteem

46. Give children more energy and increase physical activity

Cluster 3: IMPROVE DIET \& FOOD INTAKE

10. Reduce the taste preference for a high salt content in foods

14. Reduce consumption of sugary drinks

17. Reduce salt intake

21. Reduce consumption of banned and restricted foods

23. Encourage children to try a wider range of foods and new foods

59. Increase the amount of fruit juice and fruit drinks consumed by children

69. Increase consumption of oily fish

76. Increase fruit and vegetable consumption at home

81. Increase the consumption of complex carbohydrates (e.g. bread)

82. Encourage children to eat healthier packed lunches

Cluster 4: INCREASE FOOD LITERACY

29. Increase children's preferences for healthy foods

32. Encourage children to make healthier food choices outside school

44. Increase children's knowledge of healthy eating and a balanced diet

58. Help healthy foods and drinks to become the norm

85. Increase children's awareness of healthy foods

Cluster 5: IMPROVE FOOD CULTURE

12. Increase expectations among children and parents for better quality foods

13. Encourage eating to become a more enjoyable and social event

47. Encourage positive attitudes towards a healthy diet

56. Have a positive influence on family diet

62. Improve children's knowledge of food sources and production

Cluster 6: ENCOURAGE HEALTHY DIET CHOICES

16. Make food choices easier for children at school

51. Encourage children to view unhealthy foods as a treat

61. Increase peer pressure to eat healthy food

Cluster 7: IMPROVE SCHOOL MEALS \& UPTAKE

8. Improve the parents' perception of school lunches

28. Eliminate mixed health messages between the classroom and food served in the canteen

40. Improve the quality (e.g. taste and presentation) of food provided in schools

48. Reduce parental anxieties about the type of food provided in school

53. Reduce the stigma of free school meals

57. Increase purchasing pressure on parents ('pester power') to buy healthier foods

70. Increase the uptake of school lunches

Cluster 8: EDUCATIONAL BENEFITS

15. Improve the concentration and behaviour of children in class

33. Improve the diet of teachers

41. Reduce sickness absences from schools

42. Create a new school ethos about healthy lifestyles

52. Increase educational attainment

65. Increase parents' knowledge of healthy eating and a balanced diet

Cluster 9: IMPROVE LOCAL FOOD ECONOMY

24. Boost demand for local food production

30. Encourage manufacturers to make healthier products which meet the new guidelines

37. Encourage greater partnership within and between local authorities

66. Increase job satisfaction for catering staff

71. Encourage improved catering standards in other institutions, e.g. hospitals, nurseries

73. Eliminate inappropriate food subsidies in schools 

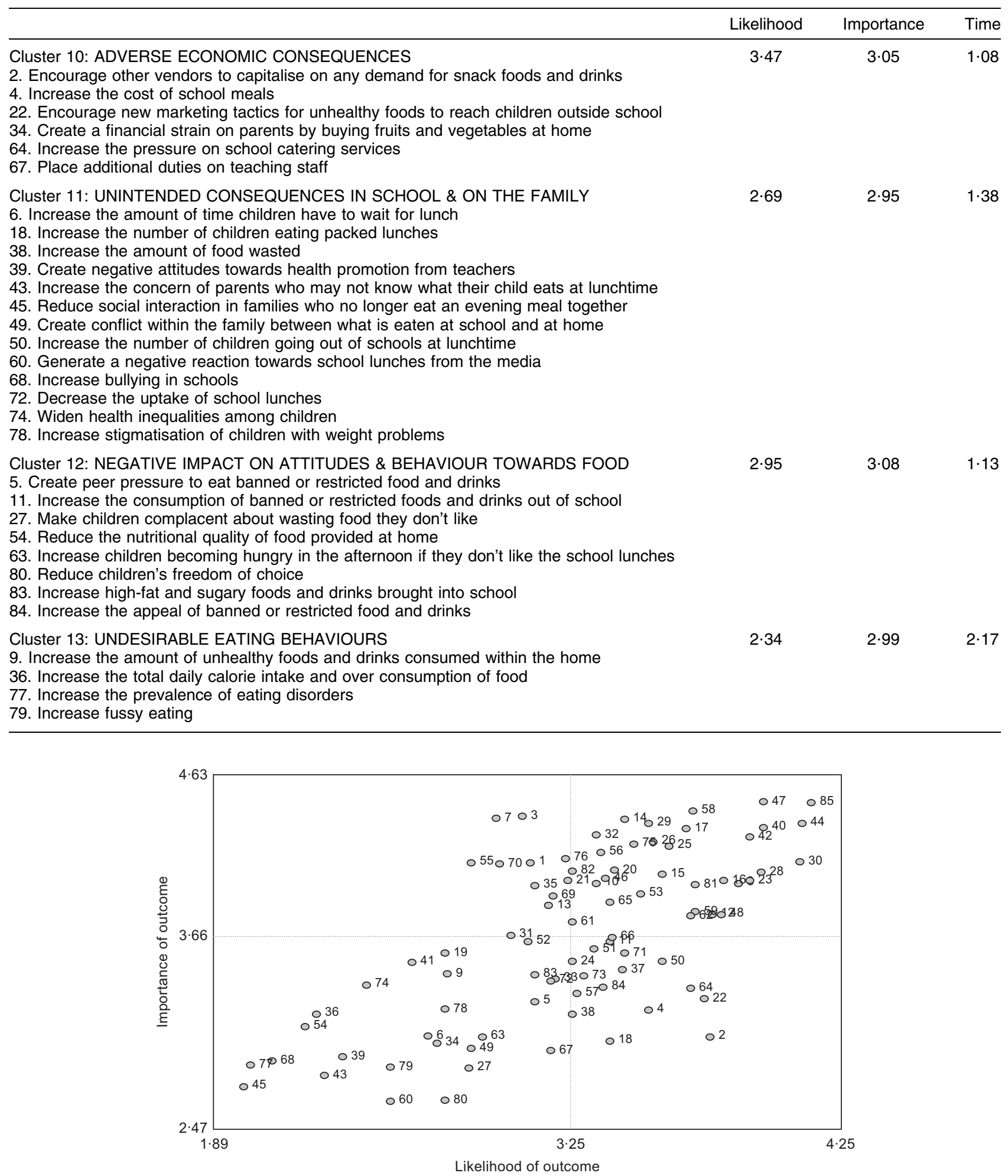

Fig. 2 The association between ratings of 'likelihood' and 'importance' for individual statements; $r=0.65$ (the number at each point relates to the statements in Table 3 )

The time to impact of the thirteen clusters provided a logical sequence of how the intervention might change behaviours, shift social norms and ultimately impact on health outcomes. The analysis revealed that the time to impact for the clusters often overlapped, because they were made up of several outcomes. For example, the 


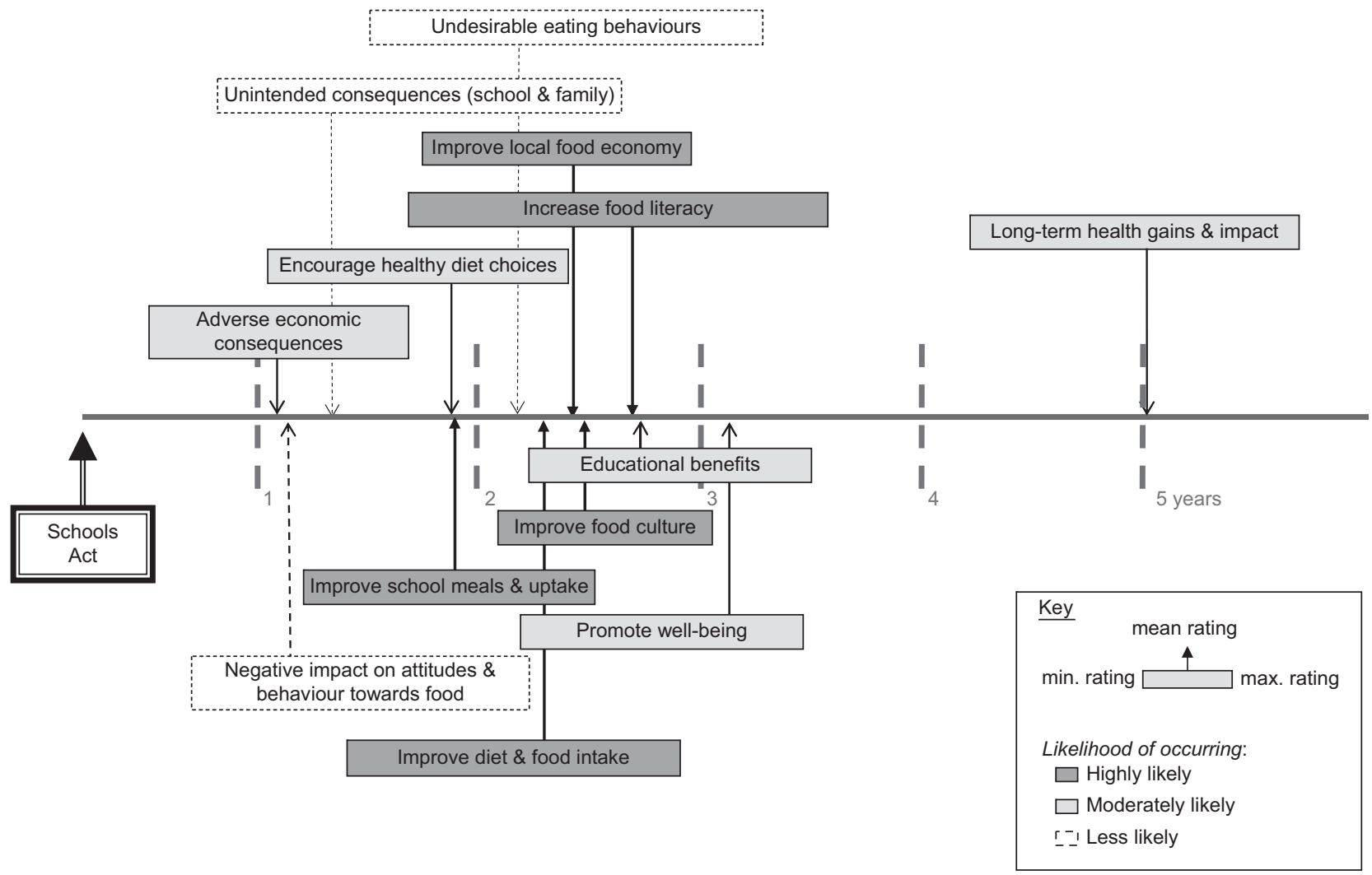

Fig. 3 Timeline for the anticipated 'time to impact' after the implementation of the Schools (Health Promotion and Nutrition) Act (Scotland) 2007

Table 4 Comparison of time to impact by stakeholder groups for each cluster

\begin{tabular}{|c|c|c|c|c|c|c|c|}
\hline \multirow[b]{2}{*}{ Cluster } & \multicolumn{2}{|c|}{ Research ( $n$ 36) } & \multicolumn{2}{|c|}{ Policy (n 19) } & \multicolumn{2}{|c|}{ Implementation (n 45) } & \multirow[b]{2}{*}{ Group differences` } \\
\hline & Mean & $95 \% \mathrm{Cl}$ & Mean & $95 \% \mathrm{Cl}$ & Mean & $95 \% \mathrm{Cl}$ & \\
\hline 1. Long-term health \& impact & $4 \cdot 81$ & $4 \cdot 47,5 \cdot 16$ & $5 \cdot 07$ & $4 \cdot 70,5 \cdot 45$ & $5 \cdot 14$ & $4 \cdot 95,5 \cdot 33$ & $P=0 \cdot 197$ \\
\hline 2. Promote well-being & $2 \cdot 62$ & $2 \cdot 19,3 \cdot 05$ & $2 \cdot 96$ & $2 \cdot 39,3.52$ & $3 \cdot 65$ & $3 \cdot 29,4 \cdot 02$ & $P=0.001^{\mathrm{a}}$ \\
\hline 3. Improve diet \& food intake & 1.93 & $1 \cdot 60,2 \cdot 27$ & $2 \cdot 13$ & $1 \cdot 78,2 \cdot 48$ & $2 \cdot 69$ & $2 \cdot 39,2 \cdot 98$ & $P=0.002^{\mathrm{a}}$ \\
\hline 4. Increase food literacy & $2 \cdot 30$ & $1 \cdot 89,2 \cdot 71$ & $2 \cdot 36$ & $1 \cdot 94,2 \cdot 77$ & 3.09 & $2 \cdot 75,3.42$ & $P=0.004^{\mathrm{a}, \mathrm{b}}$ \\
\hline 5. Improve food culture & $2 \cdot 14$ & $1 \cdot 78,2 \cdot 50$ & $2 \cdot 16$ & $1 \cdot 76,2 \cdot 57$ & $2 \cdot 82$ & $2 \cdot 52,3 \cdot 12$ & $P=0.004^{\mathrm{a}, \mathrm{b}}$ \\
\hline 6. Encourage healthy diet choices & 1.53 & $1 \cdot 16,1 \cdot 90$ & $1 \cdot 69$ & $1 \cdot 22,2 \cdot 16$ & $2 \cdot 01$ & $1 \cdot 91,2 \cdot 51$ & $P=0.011^{\mathrm{a}}$ \\
\hline 7. Improve school meals \& uptake & 1.64 & $1.31,1.96$ & $1 \cdot 42$ & $1 \cdot 09,1 \cdot 76$ & $2 \cdot 28$ & $1 \cdot 98,2 \cdot 57$ & $P=0.001^{\mathrm{a}, \mathrm{b}}$ \\
\hline 8. Educational benefits & $2 \cdot 30$ & $1 \cdot 89,2 \cdot 72$ & $2 \cdot 34$ & $1 \cdot 98,2 \cdot 71$ & $3 \cdot 14$ & $2 \cdot 83,3 \cdot 45$ & $P=0.001^{\mathrm{a}, \mathrm{b}}$ \\
\hline 9. Improve local food economy & $2 \cdot 28$ & $1 \cdot 99,2 \cdot 57$ & $1 \cdot 82$ & $1 \cdot 26,2 \cdot 38$ & $2 \cdot 79$ & $2.51,3.08$ & $P=0.001^{\mathrm{b}}$ \\
\hline 10. Adverse economic consequences & 0.96 & $0 \cdot 74,1 \cdot 19$ & 0.80 & $0.55,1.05$ & $1 \cdot 28$ & $1 \cdot 05,1 \cdot 50$ & $P=0.022^{b}$ \\
\hline 11. Unintended consequences in school \& on family & $1 \cdot 33$ & $1 \cdot 00,1 \cdot 66$ & $1 \cdot 02$ & $0.68,1.36$ & $1 \cdot 42$ & $1 \cdot 12,1 \cdot 74$ & $P=0.299$ \\
\hline $\begin{array}{l}\text { 12. Negative impact on attitudes \& behaviour } \\
\text { towards food }\end{array}$ & $0 \cdot 77$ & $0.49,1.05$ & 0.90 & $0.55,1 \cdot 24$ & $1 \cdot 44$ & $1 \cdot 12,1 \cdot 75$ & $P=0.004^{\mathrm{a}}$ \\
\hline 13. Undesirable eating behaviours & $1 \cdot 76$ & $1 \cdot 30,2 \cdot 29$ & $2 \cdot 29$ & $1 \cdot 46,3 \cdot 11$ & $2 \cdot 16$ & $1 \cdot 71,2 \cdot 60$ & $P=0.426$ \\
\hline
\end{tabular}

${ }^{*}$ Differences between groups (post hoc comparisons using Tukey's Honestly Significant Difference test): ${ }^{a}$ research $v$. implementation, ${ }^{b}$ policy $v$. implementation $(P<0.05)$.

food literacy cluster included knowledge, attitudes and food choices. Measurable changes in awareness and knowledge of a healthy diet were anticipated within 2 years, while for social norms, preferences and food choice the anticipated time was over 3 years (cluster average $2 \cdot 7$ years). The time points that emerged in this framework typically spanned several months or years.
The mean time points therefore were intended to serve as a guide to indicate when to expect change, not to identify an exact time point.

With the high prevalence of obesity among children and adults ${ }^{(29,30)}$ there is a growing pressure for policies and community interventions to tackle this major public health problem ${ }^{(31-33)}$. Although the aim would be to 
reduce the prevalence of obesity, using weight status as the primary outcome of population-based interventions, without short- and medium-term indicators, would need a very long period of follow-up to reach a conclusion about the impact of the intervention. Kropski et al. ${ }^{(34)}$ recently reviewed fourteen school-based obesity prevention programmes which used weight or BMI as a primary or secondary outcome. The review concluded that the evidence for school-based interventions was weak, but the authors stated the time required for small behavioural changes in diet or physical activity to manifest in anthropometric measures may exceed the duration of the studies reviewed'. The duration of the studies included in the review ranged from 6 months to 6 years, with the median duration of 1.8 years. This contrasts with results from our study, where it was anticipated that the earliest time to impact for a reduction in the prevalence of overweight or obesity was over 5 years. The need for short- and intermediate-term outcomes is supported by the conclusions of other reviews stating that the duration for evaluation of impact on weight or BMI for many studies is too short and that a broader range of outcomes needs to be measured ${ }^{(34,35)}$.

In general, stakeholders working in the community and involved in implementing policy in practice anticipated the time to impact would be longer than that anticipated by those working in research or policy. This difference may reflect their experiences of implementing complex policy interventions in different settings. A researcher's evidence base is likely to come from studies carried out under ideal situations where a controlled intervention is fully implemented; this is likely to be in contrast to stakeholders implementing public health policies in the 'real world' where there is less control over the intervention, which may result in only partial implementation, and other external factors ${ }^{(4,23,36,37)}$. This highlights the importance of including the expertise and experience of stakeholders, because if the evaluation timeline was based only on empirical evidence from studies carried out under ideal conditions it is likely that it would underestimate the real time to impact. Policies need time to be fully implemented and embedded in practice, before judgements about their impacts on distal outcomes such as dietary changes and health outcomes are made ${ }^{(7,11,15,16)}$.

Rating the likelihood of the outcome occurring was an important factor. It can help decide which outcome variables would be most useful to monitor and gives an indication of the size of impact of different outcomes. This can reduce the risk of measuring outcomes that may be thought to be important but unlikely to have an impact. Reducing the cost to the NHS and reducing the prevalence of type 2 diabetes among children were both rated highly on importance but low on likelihood, suggesting that these would not be good indicators to include in the evaluation of this policy. Unintended and negative consequences tended to be rated as less likely to occur than behaviours more closely associated with action of the Act. The low level of the importance given to the economic outcomes may reflect a bias towards a more health-oriented group of stakeholders.

The use of concept mapping would be considered too detailed for simple, well-controlled and well-defined interventions with a clear theory underpinning the intervention and predictable time to impact, but highly relevant for policy interventions where the theory and empirical data are often lacking. Ideally all policies should have a sound theory underpinning them ${ }^{(38)}$, but in practice this is not always the case and therefore this type of approach to evaluation is needed ${ }^{(21)}$. The increasing demand for evidence-based policy requires better evaluation of policy and, as such, appropriate methods to develop evaluations.

This method for developing an evaluation framework has strengths and limitations. A stakeholder-driven approach combines knowledge of the empirical evidence with 'real-world' perspective and experiences of working directly with the intended beneficiaries of policy interventions. This serves to give a more realistic, plausible and achievable evaluation timeline to guide data collection and the evaluation. Including this in the planning stage gives stakeholders and policy developers a better understanding of the intervention and the logical progression. One strength of the current study was that a diverse range of stakeholders participated giving different perspectives and who collectively had expert knowledge of the day-to-day schools context, research knowledge associated with health promotion, behaviour change and nutrition issues, and policy processes. Some stakeholders had little prior knowledge about the Schools Act before participating, but they were given detailed information about it before completing each stage of the process. Furthermore, no significant differences in the timeline were found between stakeholders with little or no prior knowledge of the Act before the study compared with those who had detailed prior knowledge.

A general limitation of any model developed for policy evaluation is that it assumes a stable environment, which does not take into account implementation failure. Therefore, a cautionary note has to be considered with the final timeline as it is based on the assumption that the intervention was fully implemented as intended; but in contrast to research studies this does not necessarily happen with all policy interventions. This could delay changes in the outcomes on the timeline. It was interesting to note that negative outcomes (e.g. economic, uptake of school meals) were considered to be more likely to emerge sooner than positive health or dietary change outcomes. Stakeholders expected some indicators to get worse before they got better (e.g. uptake of school meals) and this is important to note within a public policy context that is currently underpinned by tight fiscal constraints. Interestingly this trend has now been observed 
for uptake since the implementation of the Act; an initial reduction in uptake was observed in the year that changes were first made but over subsequent years there has been a recovery and increase in uptake ${ }^{(39)}$. A populated timeline of outcomes, derived from an exercise like this, may assist policy makers to make the case to continue a public health policy intervention, in challenging economic conditions, that may not yet have yielded positive health-related results but is considered likely to do so in the longer term. Conversely it can help to detect possible negative impacts (such as economic issues and implications) with a policy, especially in the early stages post implementation, and help provide a more realistic picture of the overall impact. A potential limitation of the study was that we did not include of the views of children as a stakeholder group, which could have given an additional dimension during the brainstorming. Completing the rating and sorting exercise was time-consuming, which can place a burden on stakeholders.

In summary, the purpose of the current study was to develop a timeline for evaluating nutrition-related policy interventions and it has demonstrated an approach to developing a conceptual framework. It produced a time-toimpact 'road map' connecting a broad range of outcomes to better understand the likely impact. The Schools Act provided a multilevel example of a policy intervention which not only aims to change individual behaviour through education to develop knowledge and understanding about healthy eating, but is also concerned with developing supportive organisational and cultural changes through local environmental modifications. This integrated health promotion approach can form the basis of strategies for tackling obesity through other settings such as the workplace. We believe our study offers a way of gaining a better understanding of the range of outcomes that can be affected by policy, as well as identifying potential unintended consequences. The timeline could be adapted and used as a guide for future evaluations. It offers policy makers and researchers a way of identifying possible progress towards challenging long-term public health goals, within the constraints of relatively short government policy cycles.

\section{Acknowledgements}

Sources of funding: The study was funded by the Rural and Environment Research Analysis Directorate (Scottish Government). Conflicts of interest: The authors have no conflicts of interest to declare. Author contributions: J.I.M. conceived the study and all the authors contributed to the design and interpretation of the results; J.I.M. and J.L. conducted the study and data analysis. J.I.M. was responsible for writing the manuscript and J.L., F.D., A.L., C.C. and G.M. commented on and edited the manuscript. Acknowledgements: The authors would like to thank all the stakeholders who gave their time to participate in the project.

\section{References}

1. Petticrew M, Cummins S, Ferrell C et al. (2005) Natural experiments: an underused tool for public health? Public Health 119, 751-757.

2. Foresight (2007) Tackling Obesities: Future Choices. http:// www.foresight.gov.uk/Obesity/Obesity_final/Index.html (accessed October 2007)

3. House of Commons Health Committee (2009) Health Inequalities. Third Report of Session 2008-9. vol. 1: Hc 286-1. London: The Stationery Office.

4. Pawson R \& Tilley N (1997) Realistic Evaluation. London: Sage.

5. Craig P, Dieppe P, Macintyre S et al. (2008) Developing and evaluating complex interventions: the new Medical Research Council guidance. BMJ 337, 979-983.

6. Campbell M, Fitzpatrick R, Haines A et al. (2000) Framework for design and evaluation of complex interventions to improve health. BMJ 321, 694-696.

7. Tones K (1997) Beyond the randomized controlled trial: a case for 'judicial review'. Health Educ Res 12, 161.

8. Rootman I, Goodstadt M, Potvin L et al. (2001) A framework for health promotion evaluation. In Evaluation in Health Promotion. Principles and Perspectives. WHO Regional Publications, European Series no. 92, pp. 7-38 [I Rootman, M Goodstadt, B Hyndman et al., editors]. Copenhagen: WHO Regional Office for Europe.

9. Springett J (2001) Appropriate approaches to the evaluation of health promotion. Crit Public Health 11, 139-140.

10. Bonner L (2003) Using theory-based evaluation to build evidence-based health and social care policy and practice. Crit Public Health 13, 77-92.

11. Nutbeam D (1998) Evaluating health promotion - progress, problems and solutions. Health Promot Int 13, 27-44.

12. Green J \& South J (2006) Evaluation, 1st ed. Maidenhead: Open University.

13. Hawe P, Degeling D \& Hall J (1990) Evaluating Health Promotion: A Health Worker's Guide, 1st ed. Artarmon, NSW: McLennan \& Petty.

14. Fishbein M (1996) Great expectations, or do we ask too much from community-level interventions? Am J Public Health 86, 1075-1076.

15. Hunter DJ (2009) Relationship between evidence and policy: a case of evidence-based policy or policy-based evidence? Public Health 123, 583-586.

16. Wimbush E \& Watson J (2000) An evaluation framework for health promotion: theory, quality and effectiveness. Evaluation 6, 301-321.

17. Kubisch A, Brown P, Chaskin R et al. (1997) Voices from the Field: Learning from Comprehensive Community Initiatives. Washington, DC: Aspen Institute.

18. Milstein RL \& Wetterhall SF (1999) Framework for program evaluation in public health. Centers for Disease Control and Prevention. MMWR Morb Mortal Wkly Rep 48, issue RR-11, 1-40.

19. Trochim WMK (1989) An introduction to concept mapping for planning and evaluation. Eval Program Plann 12, $1-16$.

20. Tones K \& Tilford S (2001) Health Promotion: Effectiveness, Efficiency and Equity, 3rd ed. Cheltenham: Nelson Thornes.

21. Sridharan S, Campbell B \& Zinzow H (2006) Developing a stakeholder-driven anticipated timeline of impact for evaluation of social programs. Am J Eval 27, 148-162.

22. Trochim WMK, Milstein B, Wood BJ et al. (2003) Setting objectives for community and systems change: an application of concept mapping for planning a statewide health improvement initiative. Health Promot Pract 5, 8-19. 
23. Kelly CM, Baker EA, Brownson RC et al. (2007) Translating research into practice: using concept mapping to determine locally relevant intervention strategies to increase physical activity. Eval Program Plann 30, 282-293.

24. Kraemer HC \& Winkleby MA (1997) Do we ask too much from community-level interventions or from intervention researchers? Am J Public Health 87, 1727.

25. The Scottish Government (2007) Schools (Health Promotion and Nutrition Act) (Scotland) 2007. http://www. scotland.gov.uk/Topics/Education/Schools/HLivi/foodnutrition (accessed July 2010)

26. Pandey SK \& Wright BE (2006) Connecting the dots in public management: political environment, organisational goal ambiguity, and the public manager's role ambiguity. J Public Admin Res Theory 16, 511-532.

27. Lee JW, Rainey GH \& Chun YH (2009) Of politics and purpose: political salience and goal ambiguity of US federal agencies. Public Admin 87, 457-484.

28. Kane M \& Trochim WMK (2007) Concept Mapping for Planning and Evaluation. Thousand Oaks, CA: Sage Publications, Inc.

29. Bromley C, Bradshaw P \& Given L (editors) (2009) The Scottish Health Survey 2008. Edinburgh: The Stationery Office.

30. Craig R \& Mindell J (2008) Health Survey for England 2006. vol. 2: Obesity and Other Risk Factors in Children. Leeds: The Information Centre.
31. Hill JO, Peters JC \& Wyatt HR (2007) The role of public policy in treating the epidemic of global obesity. Clin Pharmacol Ther 81, 772-775.

32. Magnusson RS (2008) What's law got to do with it? Part 1: A framework for obesity prevention. Aust $N Z$ Health Policy 5, 10.

33. Swinburn BA (2008) Obesity prevention: the role of policies, laws and regulations. Aust N Z Health Policy 5, 12.

34. Kropski JA, Keckley PH \& Jensen GL (2008) School-based obesity prevention programs: an evidence-based review. Obesity (Silver Spring) 16, 1009-1018.

35. Summerbell CD, Waters E, Edmunds LD et al. (2005) Interventions for preventing obesity in children. Cochrane Database Syst Rev issue 3, CD001871.

36. Judge K \& Bauld L (2001) Strong theory, flexible methods: evaluating complex community-based initiatives. Crit Public Health 11, 20-38.

37. Leviton LC (2008) Children's healthy weight and the school environment. Ann Am Acad Pol Soc Sci 615, 38-55.

38. Kelly MP, Stewart E, Morgan A et al. (2009) A conceptual framework for public health: NICE's emerging approach. Public Health 123, e14-e20.

39. The Scottish Government (2009) School Meals in Scotland, 2009. Table 3: Pupils taking school meals (free or paid for) by sector, 2000 to 2009. http://www.scotland.gov.uk/ Publications/2009/06/22104701/5 (accessed July 2010). 